Mariana Matera VERAS ${ }^{1}$ Maria Angélica MIGLINO' Zenon SILVA²

Correspondência para: MARIAANGÉLICAMIGLINO Departamento de Cirurgia Faculdade de Medicina Veterinária Universidade de São Paulo Av. Prof. Dr. Orlando Marques de Paiva, 87 05508-000 - São Paulo-SP miglino@usp.br

Recebido para publicação: 15/12/2004 Aprovado para publicação: 18/12/2006

\title{
Morfologia do aparelho reprodutor em fêmeas de bugio marrom (Alouatta guariba clamitans)
}

1 - Departamento de Cirurgia da Faculdade de Medicina Veterinária e Zootecnia da Universidade de São Paulo, São Paulo - SP

2 - Departamento de Anatomia da Faculdade de Medicina da Universidade Federal de Uberlândia, Uberlândia - MG

\section{Resumo}

Estudaram-se a morfologia externa, a topografia e a biometria do aparelho reprodutor (ovários, útero, vagina e as tubas uterinas) de seis fêmeas adultas de bugio marrom (Alouatta guariba clamitans). Notou-se que os ovários apresentaram-se grandes e elipsóides, com superfície lisa ou irregular. As tubas uterinas mostraram-se longas, convolutas e com fimbrias bem desenvolvidas e abundantes. O útero é simples e marcado por uma cérvix longa, bem desenvolvida e um fundo globoso. A vagina mostra-se como um canal alongado, de paredes finas e revestido por uma mucosa com pregas longitudinais; o orifício externo da uretra é marcado por uma papila uretral bilobada.

\section{Introdução}

Os bugios estão incluídos no grupo dos Platyrrhini (primatas neotropicais) e pertencem à família Atelidae, gênero Alouatta, cujos representantes são estritamente americanos. Outros representantes da família Atelidae são o macaco-aranha (gênero Ateles), o macaco-barrigudo ou mono (gênero Lagothrix) e o muriqui (gênero Brachyteles) ${ }^{1}$. De acordo com a Lista Oficial da Fauna Brasileira Ameaçada de Extinção (2002) das 133 espécies e subespécies de primatas brasileiros 24\% encontram-se ameaçadas ou quase ameaçadas. Os bugios marrons (Alouatta guariba clamitans) estão classificados como quase ameaçados embora existam muitos trabalhos sobre o comportamento desta espécie, pouco se sabe sobre a fisiologia reprodutiva e sobre a morfologia dos órgãos reprodutores. Agrava a situação o fato de a espécie apresentar dificuldades para se reproduzir em cativeiro ${ }^{2}$. Sabe-se que muitas vezes uma espécie encontra-se seriamente ameaçada e pouco se sabe sobre seus aspectos reprodutivos, fazendo com que o uso de técnicas de reprodução assistida para sua conservação torne-se difícil ${ }^{3}$. Assim, o presente estudo teve como objetivo examinar e descrever a morfologia macroscópica do aparelho reprodutor de fêmeas de bugio marrom, de modo a estender o conhecimento anatômico prévio e fornecer dados para a adequação de técnicas de reprodução assistida.

\section{Material e Método}

Um total de seis fêmeas adultas foi utilizado neste estudo. Três cadáveres de fêmeas adultas que vieram a óbito, foram cedidos pelo Depave 3-SP (Departamento de Parques e Áreas Verdes). Outros três cadáveres foram cedidos pelo CEPESBIIndaial-SC (Centro Pesquisas Biológicas), onde é desenvolvido o Projeto Bugio. Para se entender as relações entre os órgãos e sua topografia foi realizada autópsia em 4 animais, previamente fixados, por perfusão de solução de formalina 10\% pela artéria femoral. A vagina, o útero, a cérvix, as tubas e os ovários foram examinados após dissecação e secção. As mensurações dos órgãos (útero, vagina, ovários e tuba) foram obtidas com auxílio de um paquímetro digital e régua calibrada.

Para os ovários foram obtidas as seguintes medidas: largura - da face lateral à 
face medial; comprimento - da extremidada tubal à extremidade uterina; e altura - da margem mesovárica à margem livre. Quando presente a medida do corpo lúteo foi inserida. As tubas uterinas foram dissecadas e seu comprimento foi obtido do óstio abdominal ao óstio uterino, acompanhando seus contornos com um fio de náilon. O comprimento do útero foi obtido a partir do fundo até a junção com vagina, enquanto o comprimento da vagina compreendia a distância do vestíbulo até a junção com o útero.

A nomenclatura adotada foi baseada no International Committee on Veterinary Gross Anatomical Nomenclature4.

\section{Resultados}

Como em todos os primatas, os órgãos genitais consistiam de dois ovários, duas tubas uterinas, útero e vagina (Figura 1). Localizada na pelve, a vagina apresentase como um longo canal, de paredes finas, comprimido dorsoventralmente; na porção cranial sua parede está aplicada ao redor da cérvix uterina formando uma fenda circular (fórnices vaginais). O comprimento da cérvix uterina variou de 23 a $38,5 \mathrm{~mm}$ com média de 33,57 mm (Tabela 1). A dissecação da vagina revelou uma mucosa com pregas longitudinais em toda sua extensão. Incisando-se longitudinalmente o canal vaginal, pôde-se visualizar um estreitamento,

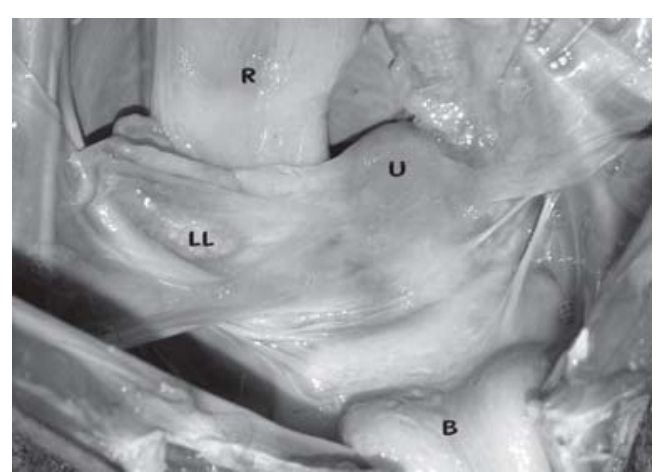

Figura 1 - Dissecação longitudinal da vulva ao útero. Observar os ovários $(\mathrm{Ov})$, fimbrias $(\mathrm{F})$, útero $(\mathrm{U})$, vagina $(\mathrm{V})$, vulva $(\mathrm{Vv}) \mathrm{e}$ a vesícula urinária $(\mathrm{Vu})$ na altura do óstio externo da uretra, formando um ístimo, que divide a vagina em duas porções. O óstio externo da uretra abre-se na região medial da parede ventral em uma papila distinta, proeminente e bilobada (Figura 2).

Os ovários são duas estruturas bilaterais elipsóides situadas em cada lado do útero em relação à parede lateral da pelve, fixados posteriormente ao ligamento largo e inferiormente às tubas uterinas e com superfície lisa ou granulosa. Estão situados em uma depressão delimitada pelo peritônio, chamada fossa ovárica. Cada ovário apresenta uma face medial e outra lateral, uma extremidade tubária e outra uterina e uma borda livre e outra mesovariana. A borda mesovariana é retilínea e está fixa ao ligamento largo por uma pequena prega, o mesovário. A borda livre é côncava e é parcialmente recoberta pela extremidade fimbriada da tuba uterina. A extremidade uterina é mais aguda e está direcionada em relação ao assoalho da pelve. O ligamento próprio do ovário é espesso e curto, preso a extremidade uterina mantém os ovários bem próximos ao útero. A extremidade oposta recebe o ligamento suspensor do ovário que se prende à parede lateral da pelve na altura. A extremidade tubárica está fixa pela fimbria ovárica (Fig.3) e pelo ligamento suspensor do ovário, o qual se inicia na parede lateral da pelve pósterolateralmente ao nível da última flexura do

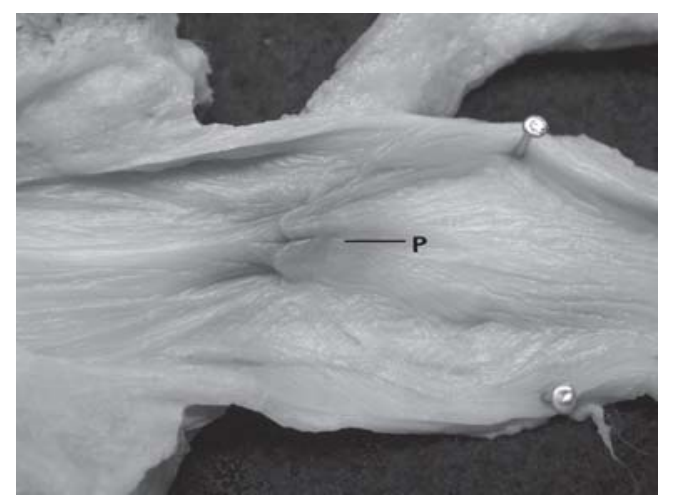

Figura 2 - Fotografia da parede ventral da vagina, observar a papila uretral bilobada e as pregas da mucosa 


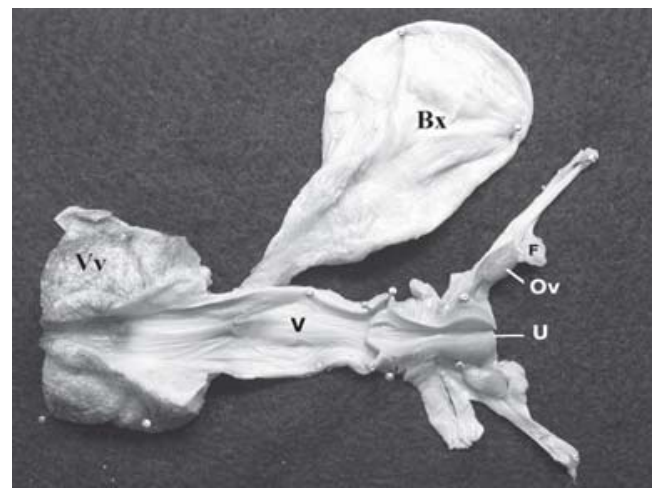

Figura 3 - Vista anatômicaduranteaautópsia. A sínfise púbicafoi aberta e a vesícula urinária deslocada para expor melhor os órgãos: reto $(R)$, vesícula urinária $(V)$, útero $(U)$ e ligamento largo (LL)

cólon sigmóide e das vértebras L4 e L5, descendo obliquamente de lateral para medial terminando no ovário. Nos animais estudados não foi notado acúmulo de tecido adiposo no mesovário (Tabela 1).

A tuba uterina é um órgão tubular muito fino e convoluto. A partir do ângulo superolateral do útero dirige-se, ao longo a margem superior do ligamento largo, até a extremidade tubal do ovário; curvando-se craniocaudalmente sobre ele. Não há distinção evidente entre infundíbulo, ampola e ístimo. As fímbrias que margeiam o infundíbulo são longas; a fimbria ovariana fixa-se ao pólo tubal do ovário. Notou-se que o comprimento médio para tuba direita era de $28,07 \mathrm{~mm}$ e da tuba esquerda de 29 $\mathrm{mm}$. Os valores dos comprimentos das tubas uterinas estão representados na Tabela 1 .

O útero é simples e alongado, caracterizado por um fundo globóide e uma cérvix marcadamente longa, que se projeta

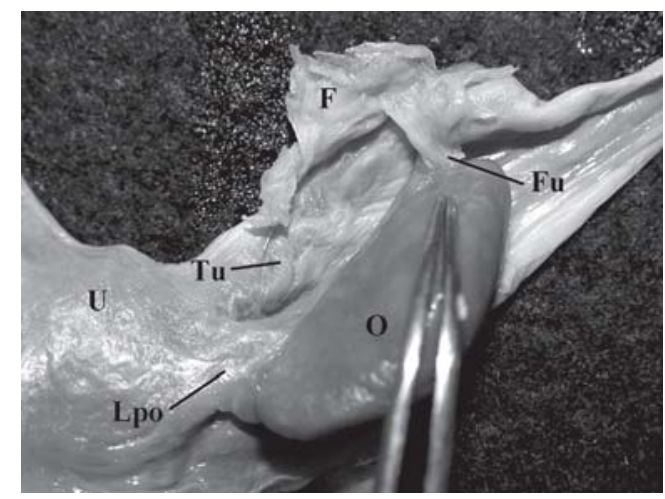

Figura 4 - Fotografia em maior aumento em relação à figura 1 para mostrar a relação entre o ovário $(\mathrm{O})$, tuba uterina $(\mathrm{Tu})$, útero (U) fímbria ovariana (Fo) eligamento próprio do ovário (Lpo)

no canal vaginal. Tanto a superfície ventral quanto a dorsal são recobertas pelo por peritônio. As reflexões do peritônio na altura da porção cervical, sobre a face ventral do reto e sobre a face dorsal da bexiga, formam, respectivamente, as escavações retouterina e vesicouterina. O útero não apresenta retoflexão, nem anteflexão e devido ao grande desenvolvimento do ceco está ligeiramente deslocado para o lado esquerdo. Os ligamentos de sustentação do útero incluem os ligamentos úterossacrais, redondo, largo e, em menor instância, os ligamentos útero-ovarianos. O ligamento largo é delicado e acompanha a borda do corpo do útero como uma membrana e se fusiona na região cervical. O ligamento redondo, recoberto pelo ligamento largo, fixa-se próximo à borda lateral do útero logo abaixo da inserção das tubas uterinas e parte em direção à parede lateral da pelve, atravessa a região inguinal e termina no lábio

Tabela 1 - Dados métricos $(\mathrm{mm})$ referentes à largura, comprimento e altura dos ovários e comprimento do útero, cérvix uterina em fêmeas adultas de bugio marrom, São Paulo - 2003

\begin{tabular}{|c|c|c|c|c|c|c|c|c|c|c|c|}
\hline \multirow{2}{*}{ Animal } & \multicolumn{3}{|c|}{ Ovário direito } & \multicolumn{3}{|c|}{ Ovário esquerdo } & \multicolumn{2}{|c|}{ Tuba uterina } & \multirow[t]{2}{*}{ Útero } & \multirow[t]{2}{*}{ Cérvix } & \multirow[t]{2}{*}{ Vagina } \\
\hline & Largura & Comprimento & Altura & Largura & Comprimento & Altura & Direita & Esquerda & & & \\
\hline 1 & 5,8 & 7,4 & 8,2 & 5,2 & 10,7 & 7,9 & 20,9 & 21,5 & 30,9 & 12 & 23 \\
\hline 2 & 5,4 & 9,3 & 6,7 & 5,6 & 9,1 & 5,3 & 26,5 & 27,2 & 27,3 & 9,5 & 24,5 \\
\hline 3 & 2,7 & 9 & 5,5 & 3,8 & 9,2 & 4,9 & 22,9 & 23,2 & 25,7 & 15,9 & 30,4 \\
\hline 4 & 7,3 & 13,2 & 8,4 & 6,2 & 8,2 & 7,5 & 24 & 29 & 28,3 & 11,1 & 42 \\
\hline 5 & 6,5 & 20 & 5,8 & 8 & 21 & 6 & 40,1 & 38 & 25 & 8 & 43 \\
\hline 6 & 6,5 & 15 & 4 & 6 & 12 & 4,5 & 34 & 35,1 & 32,5 & 15 & 38,5 \\
\hline
\end{tabular}


Tabela 2- Sumário estatístico dos dados métricos $(\mathrm{mm})$ referentes à largura, comprimento e altura dos ovários e ao comprimento das tubas uterinas, utero, cérvix e vagina, em fêmas de bugio marron, São Paulo- 2003

\begin{tabular}{|c|c|c|c|c|c|c|c|c|c|c|c|}
\hline dnt & \multicolumn{3}{|c|}{ Ovário direito } & \multicolumn{3}{|c|}{ Ovário esquerdo } & \multicolumn{2}{|c|}{ Tuba uterina } & \multirow[t]{2}{*}{ Útero } & \multirow[t]{2}{*}{ Cérvix } & \multirow[t]{2}{*}{ Vagina } \\
\hline m & Largura & Comprimento & Altura & Largura & Comprimento & Altura & Direita & Esquerda & & & \\
\hline Média & 5,7 & 12,32 & 6,43 & 5,8 & 11,7 & 6,02 & 28,07 & 29 & 28,28 & 11,92 & 33,57 \\
\hline $\begin{array}{l}\text { Valor } \\
\text { mínimo }\end{array}$ & 2,7 & 7,4 & 4 & 3,8 & 8,2 & 4,5 & 20,9 & 21,5 & 25 & 8 & 23 \\
\hline $\begin{array}{l}\text { Valor } \\
\text { máximo }\end{array}$ & 7,3 & 20 & 8,4 & 8 & 21 & 7,9 & 40,1 & 38 & 32,5 & 15,9 & 38,5 \\
\hline $\begin{array}{l}\text { Desvio } \\
\text { Padrão }\end{array}$ & 1,61 & 4,72 & 1,69 & 1,37 & 4,75 & 1,4 & 7,44 & 6,5 & 2,93 & 3,07 & 8,81 \\
\hline
\end{tabular}

maior. Tanto o corpo quanto o fundo do útero estão localizados inferiormente à borda cranial da sínfise pubiana (Figura 4). Não há constrições demarcando o início do segmento cervical. Em secção a cérvix pode ser distinguida do corpo uterino. O canal endocervical é muito constricto e sua mucosa é pregueada. A abertura do canal tem a forma de uma fenda transversa. $\mathrm{O}$ lúmen uterino é reduzido e quando seccionado coronalmente a cavidade tem aparência de um triângulo invertido. $\mathrm{O}$ comprimento médio do útero foi de 28,28 $\mathrm{mm}$ e do segmento cervical de $11,92 \mathrm{~mm}$ (Tabela 2).

\section{Discussão}

Analisando-se a topografia e a morfologia macroscópica dos órgãos reprodutores das fêmeas de bugio marrom (Alouatta guariba clamitans), notou-se que assemelham-se àquelas descritas em primatas. Há, entretanto, particularidades entre os diversos gêneros e espécies, qual consideramos, diante da necessidade de esclarecer pormenores relativos à morfologia reprodutiva. Estudos sobre a anatomia de primatas neotropicais são raros e muitas vezes não atuais. A literatura trata, na maioria das vezes, de primatas utilizados em pesquisas biomédicas como o Aotus sp. e Saimiri sp 5 . Com relação aos ligamentos ovarianos e uterinos e a topografia dos órgãos reprodutores internos, os achados deste estudo se aproximam com as descrições para Lagothrix ${ }^{6}$, Aotus ${ }^{7}$ e para outros Platirrbini $^{8,9}$. Porém, em Saimiri ${ }^{8,9}$ foram encontrados ovários dispostos longitudinalmente, como em animais quadrúpedes, e o ligamento próprio do ovário era longo quando comparado ao das fêmeas desta amostra. Não foram verificados acúmulos de tecido adiposo no mesossalpíngeo, ao contrário do que ocorrem em chimpanzés e orangotangos ${ }^{8,9}$. Não há relato de acúmulo em outros Platyrrbin 8 . O útero de bugios é simples e alongado e apresenta a porção cervical bem desenvolvida que ao se projetar na vagina forma uma fenda circular (fórnix vaginal). Também, em Ateles e Cebus a cérvix exibe esta configuração ${ }^{8}$. Já em Aotus $^{7}$, Saimiri ${ }^{10}$ e Lagothrix ${ }^{6}$ tanto a cérvix quanto a forma do fundo uterino exibem conformações distintas daquelas encontradas nas fêmeas de bugio. Embora a conformação da cérvix seja distinta entre Lagothrix e Alouatta a mucosa cervical nestes dois gêneros apresenta pregas longitudinais visíveis macroscopicamente. A descrição da forma dos ovários de Alonatta caraya $^{11}$ (bugio negro) como estruturas globosas, diverge da forma alongada e elipsóide que foi constata neste estudo. Nos bugios, as pregas da mucosa vaginal estendem-se por todo o canal vaginal; em Cebus ${ }^{9}$, porém, estas pregas desaparecem na região próxima à cérvix. Assim, como em Callthrix jacchus $^{13}$, pôde-se notar uma divisão do canal vaginal em duas porções, uma superior e outra inferior, por um estreitamento na região superior, a papila uretral. As tubas uterinas apresentaram o lúmen muito estreito e mostram-se espiraladas, porém esta característica não é constante entre Platirrhyni, pois em Lagothrix ${ }^{5}$, 
Callithrix ${ }^{13,14}$ e Saimiri ${ }^{8}$ elas são quase lineares. Não foi possível distinguir macroscopicamente as diferentes regiões da tuba uterina, outros autores não relataram diferenças para estes aspectos em Lagothrix ${ }^{6}$, apenas foram apontadas constrições nas regiões do ístimo e da porção intramural. Andersen ${ }^{15}$ que estudou um exemplar de Alouatta palliata também não notou distinções, somente salientou que o lúmen apresentava-se reduzido e que não havia um esfíncter na porção intramural, assim como nossos resultados mostraram.

\section{Conclusões}

Os ovários de fêmeas de bugio são grandes, compactos e elipsóides e ligam-se ao útero por um espesso e curto ligamento próprio do ovário e à parede lateral da pelve por um tênue ligamento suspensor do ovário.

As tubas uterinas são finas e convolutas: apresentam infundíbulo bem fimbriado. A fimbria ovárica está presente e a fimbria uterina ausente. Não foi possível a distinção macroscópica entre infindúbulo, ampola e ístimo.

A vagina é um canal longo de paredes finas, que se apresenta dividida por uma constrição do canal, em duas porções.

O útero é simples e alongado, com uma porção cervical bem desenvolvida e fundo globoso. Não apresenta anteflexão, nem retoflexão. Está localizado inferiormente à sínfise púbica, relacionando-se dorsalmente com o reto, ventralmente com a bexiga urinária e ceco e lateralmente com as tubas uterinas e ovários.

\section{Morphology of the female reproductive tract of the brown howler monkey (Alouatta guariba clamitans)}

\section{Abstract}

It was studied the external morphology, topography and the measures of the reproductive tract (ovaries, uterus, vagina and uterine tube) in six howler monkey females (Alouatta guariba clamitans). The ovaries are large and ellipsoid, with a smooth or irregular surface. The uterine tubes are long and convoluted, with well-developed and abundant fimbrias. The uterus is simple and characterized by a long and welldeveloped cervix and a globular fundic portion. The vagina is a long chamber with thin walls covered with a folded mucosa. The urethra opens in a distinct bilobate papilla within the ventral vagina wall.

\section{Referências}

1 RYLANDS, B. A.; SCHNEIDER, H.; LANGGUTH, A.; MITTERMEIER, R. A.; GROVES, C. P.; RODRIGUEZ-LUNA, E. An assessment of the diversity of new world primates. Neotropical primates, v. $8, n$. 2, p. $61-93,2000$

2 CROCKETT, C. M. Conservation biology of the genus Alouatta. International Journal of Primatology, v. 19, n. 3, p. 549-578, 1998.

3 WILDT, reproductive research in conservation biology: priorities and avenues for support. Journal of Zoo and Wildlife Medicine, v. 4, n. 20, p. 391-395, 1989

4 INTERNATIONAL COMMITTEE ON VETERINARY GROSS ANATOMICAL NOMENCLATURE; Nomina Anatomica Veterinaria. 4th ed. Ithaca: World Association on Veterinary Anatomists, 1994
5 GIBBSON, S. Neotropical primates in biomedical research. In: MEETING OF THE AMERICAN SOCIETY OF PRIMATOLOGIST (ASP), 27, 2004, Proceedings.. Madison: University of Wisconsin, USA. Oral presentation, session 19, 2004. < http://www.asp.org/ asp2004/index.htm >

6 HILL, W. C. O. Observations on the genitalia of the wolly monkey (Lagothrix). Proceedings of Zoological Society London., v. 122, p. 973-984, 1953.

7 HERTIG, A. T.; BARTON, B. R.; MACKEY, J. J. The female genital tract of the owl monkey (Aotus trivirgatus) with special reference to the ovary. Laboratory Animal Science, v. 26, n. 6, p.1041-1067, 1976.

8 WISLOCKI, G. B. On the female reproductive tract of the gorilla, with comparison of that of others primates. Contribution to Embryology Carnegie Institute., v. 23, p. $163-204,1932$.

9 HILL, W. C. O. Primates: comparative anatomy and 
taxonomy. Edinburgh: University Press, 1957, v.5.

10 COLBORN, G. L.; BO, W. J.; LANG, C. M. Observations on the Cervix uteri of the squirrel monkey. Journal of Morphology, v. 122, p.81-88, 1967.

11 CLAVER, J. A.; VON LAWZEWITSCH, I; COLILLAS, O. J. Microscopic anatomy of the ovary of Alouatta caraya. Primates, v. 25, n. 3, p.362-371, 1984.

13 CUI, KE-HUI; MATTHEWS, C. Anatomy of the adult female common marmoset (Callithrix jacchus) reproductive system. Journal of Anatomy, v. 185, p. 481-486, 1994

14 BEATTIE, J. The anatomy of the common marmoset. Proceedings of Zoological Society London. v. 1927, p. 593-718, 1927.

15 ANDERSEN, D. H. Comparative anatomy of the tubo uterine junction. Histology and physiology in the saw. American Journal of Anatomy, v. 42, p. 255-305, 1928. 